\title{
Broad line region and black hole mass of PKS 1510-089 from spectroscopic reverberation mapping ${ }^{\star}$
}

\begin{abstract}
Suvendu Rakshit
Finnish Centre for Astronomy with ESO (FINCA), University of Turku, Vesilinnantie 5, 20014 Quantum, Finland e-mail: suvenduat@gmail.com

Received 1 May 2020 / Accepted 14 July 2020

ABSTRACT

Reverberation results of the flat spectrum radio quasar PKS 1510-089 from 8.5 years of spectroscopic monitoring carried out at Steward Observatory over nine observing seasons between December 2008 and June 2017 are presented. Optical spectra show strong $\mathrm{H} \beta, \mathrm{H} \gamma$, and $\mathrm{Fe}$ II emission lines overlying on a blue continuum. All the continuum and emission line light curves show significant variability with fractional root-mean-square variations of $37.30 \pm 0.06 \%\left(f_{5100}\right), 11.88 \pm 0.29 \%(\mathrm{H} \beta)$, and $9.61 \pm 0.71 \%(\mathrm{H} \gamma)$; however, along with thermal radiation from the accretion disk, non-thermal emission from the jet also contributes to $f_{5100}$. Several methods of time series analysis (ICCF, DCF, von Neumann, Bartels, JAVELIN, $\chi^{2}$ ) are used to measure the lag between the continuum and line light curves. The observed frame broad line region size is found to be $61.1_{-3.2}^{+4.0}\left(64.7_{-10.6}^{+27.1}\right)$ light-days for $\mathrm{H} \beta(\mathrm{H} \gamma)$. Using the $\sigma_{\text {line }}$ of $1262 \pm 247 \mathrm{~km} \mathrm{~s}^{-1}$ measured from the root-mean-square spectrum, the black hole mass of PKS 1510-089 is estimated to be $5.71_{-0.58}^{+0.62} \times 10^{7} M_{\odot}$.
\end{abstract}

Key words. galaxies: active - galaxies: nuclei - galaxies: individual: PKS 1510-089 - quasars: supermassive black holes techniques: spectroscopic

\section{Introduction}

Active galactic nuclei (AGNs) are powered by the accretion of matter on a central supermassive black hole surrounded by an accretion disk and a broad line region (BLR; see Urry \& Padovani 1995). The BLR is photo-ionized by the UV and optical photons from the accretion disk emitting broad emission lines of full width at half maximum (FWHM; $10^{3}-10^{5} \mathrm{~km} \mathrm{~s}^{-1}$ ), which are detected through optical spectroscopy. The mass of the black hole is found to be strongly correlated with the host galaxy properties, suggesting the co-evolution of the black hole and the host galaxy (Kormendy \& Ho 2013). However, the accurate measurement of black hole masses is crucial. Black hole masses can be dynamically measured in the nearby galaxy using stars and gases; however, this is extremely challenging for AGNs beyond the local volume. More challenging still is the measurement of black hole masses in radio-loud AGNs, since their optical emission is dominated by the non-thermal emission from their relativistic jets, which are aligned close to the observer.

The quasar PKS 1510-089 is a well-studied flat spectrum radio quasar (FSRQ) located at a redshift $z=0.361$ (Thompson et al. 1990). Its optical spectrum shows broad emission lines with a blue continuum (Tadhunter et al. 1993). The broad-band spectral energy distribution (SED) of FSRQs has a double hump structure due to the combined effect of the thermal emission from the accretion disk peaking at UV and optical, and non-thermal emission from the jets (e.g., Abdo et al. 2010b).

* Full Table 1 is only available at the CDS via anonymous ftp to cdsarc.u-strasbg.fr (130.79.128.5) or via http://cdsarc. u-strasbg.fr/viz-bin/cat/J/A+A/642/A59
The low energy (radio to X-rays) is dominated by the optically thin synchrotron emission from relativistic electrons from the jet, while the high energy peak (X-ray to $\gamma$-ray) could be due to an inverse Compton process, where the seed photons originate from the BLR (Sikora et al. 1994). Similar to other radio-loud AGNs, PKS 1510-089 shows strong flux variation across the entire electromagnetic spectrum from radio to $\gamma$ ray (e.g., Malkan \& Moore 1986; Tavecchio et al. 2000; Bach et al. 2007; Abdo et al. 2010a; Marscher et al. 2010; Orienti et al. 2013; H.E.S.S. Collaboration 2013; Kushwaha et al. 2016; Beaklini et al. 2017; Castignani et al. 2017; Prince et al. 2019). However, the BLR of PKS 1510-089 remains poorly studied due to its strong radio emission, although the size of its BLR is an important model parameter in the multi-band SED fitting. Moreover, the mass of the black hole powering PKS 1510-089 remains highly uncertain (Abdo et al. 2010a).

Reverberation mapping (RM; Blandford \& McKee 1982; Peterson 1993) is a reliable tool to estimate the size of the BLR and the black hole mass through spectroscopic monitoring. It has so far provided BLR sizes for more than 100 objects (Wandel et al. 1999; Kaspi et al. 2000; Peterson et al. 2004; Bentz et al. 2009, 2013; Shen et al. 2016; Grier et al. 2017; Park et al. 2017a; Du et al. 2016a, 2018; Rakshit et al. 2019; Cho et al. 2020), allowing us to establish a relation between the size of the BLR and monochromatic luminosity (Bentz et al. 2013; Du \& Wang 2019). Recently, RM studies of a few FSRQs have been performed using multi-year monitoring data. For example, Zhang et al. (2019) performed a reverberation study of FSRQ 3C273 using ten years of Steward Observatory monitoring data, which provide a highly reliable measurements of BLR size. Nalewajko et al. (2019) performed Mg II reverberation studies of FSRQ 3C 454.3 using the Steward Observatory monitoring 
data and Zajaček et al. (2020) measured the Mg II lag of HE 0413-4031 using SALT monitoring data.

As a part of the optical spectropolarimetric monitoring program of $\gamma$-ray emitting blazars, PKS 1510-089 has been observed from Steward Observatory since 2008, with a median time sampling of $\sim 10$ days (Smith et al. 2009). In this paper, I analyze $\sim 8.5$ years (from a total of nine observing seasons) of long optical spectroscopic data obtained from Steward Observatory. The optical spectrum shows a blue continuum and the presence of strong Balmer lines $(\mathrm{H} \beta$ and $\mathrm{H} \gamma)$, as well as Fe II emission. Each Balmer line shows flux variability. I performed cross-correlation analysis to estimate the size of the BLR and the black hole mass. This is the first reverberation-based black hole mass estimate of PKS 1510-089. In Sect. 2 I describe the data analysis, and in Sect. 3 I present the result of the spectral analysis. I briefly discuss the result in Sect. 4 and conclude in Sect. 5 .

\section{Data}

\subsection{Optical data}

For this work, optical photometry and spectroscopic data from the Steward Observatory spectropolarimetric monitoring project $^{1}$, a support program for the Fermi Gamma-Ray Space Telescope, was used. Observations were carried out using the spectrophotometric instrument SPOL (Schmidt et al. 1992) with a $600 \mathrm{~mm}^{-1}$ grating, which provides a wavelength coverage of 4000-7550 $\AA$ and a spectral resolution of 15-25 $\AA$ depending on the slit width (Smith et al. 2009). Observations were performed using the $2.3 \mathrm{~m}$ Bok Telescope on Kitt Peak and the $1.54 \mathrm{~m}$ Kuiper Telescope on Mount Bigelow in Arizona. Details regarding observations and data reduction are given in Smith et al. (2009). In short, differential photometry using a standard field star was preformed to calibrate photometric magnitudes. A total of $363 \mathrm{~V}$-band photometric observations carried out between December 2008 and July 2017 were used in the work. Spectra were flux-calibrated using the average sensitivity function, which was derived from multiple observations of several spectrophotometric standard stars throughout an observing campaign. Final flux calibrations were performed, rescaling the spectrum from a given night to match the synthetic $V$-band photometry of that night (see Smith et al. 2009). Therefore, a total of 341 photometrically calibrated spectra obtained between December 2008 and June 2017 were downloaded from the Steward Observatory database and used in this work.

\subsection{Gamma-ray and radio data}

The $\gamma$-ray data were collected from the publicly available database of the Large Area Telescope (LAT) on board the Fermi GammaRay Space Telescope (Abdo et al. 2009) between December 2008 and June 2017 and within the energy range of $100 \mathrm{MeV}$ to $300 \mathrm{GeV}$. For data analysis, the Fermi Science Tool version v10r0p5 and the publicly available FERMIPY package (Wood et al. 2017) were used. The data sets within $15^{\circ}$ of the region of interest and a zenith angle cut of more than $90^{\circ}$ were considered to avoid background contamination. The instrument response function P8R2_SOURCE_V6, the isotropic background model iso_P8R2_SOURCE_V6_v06.txt, and the Galactic diffuse model gll_iem_v06.fit were also used. The analysis was performed using the maximum likelihood method ("gtlike") with the criteria

1 http://james.as.arizona.edu/ psmith/Fermi/
“(DATA_QUAL > 0)\&\&(LAT_CONFIG==1)", and the monthly binned light curve was generated.

The $15 \mathrm{GHz}$ radio data observed using the $40 \mathrm{~m}$ Telescope at the Owens Valley Radio Observatory (OVRO) were also collected. The data were obtained as part of an observation program supporting the Fermi Gamma-Ray Space Telescope and have a time sampling of about twice per week (Richards et al. 2011).

\section{Result and analysis}

\subsection{Optical spectral decomposition}

Multi-component spectral analysis was performed to obtain spectral information from each nightly spectrum. First, each spectrum was corrected for Galactic extinction using $E(B-V)=$ 0.09 (Schlafly \& Finkbeiner 2011) and the Milky Way extinction law with $R_{V}=3.1$ from Cardelli et al. (1989). Then spectra were brought to the rest-frame using $z=0.361$. Finally, a multicomponent spectral analysis was performed, as done in previous works (e.g., Rakshit \& Woo 2018; Rakshit et al. 2019).

In the spectral analysis method, the continuum was first modeled with a single power-law in the form of $f_{\lambda}=\beta \lambda^{\alpha}$. Additionally, to model Fe II emission, an Fe II template from Kovačević et al. (2010) was used as it provides accurate fitting of blended Fe II emission lines (e.g., Park et al. 2017b). During this step, all the broad and narrow emission lines were masked out. Using the IDL fitting package MPFIT $^{2}$ (Markwardt 2009), a nonlinear Levenberg-Marquardt least-squares minimization was performed to find the best-fit continuum model. Then the best-fit continuum model was subtracted from each spectrum and the residual spectrum was used to model emission lines.

The $\mathrm{H} \beta$ emission line complex was modeled in the wavelength range of $4740 \AA-5050 \AA$, where a sixth-order Gauss-Hermite $(\mathrm{GH})$ series was used to model the $\mathrm{H} \beta$ broad component, and a Gaussian was used to model the narrow $\mathrm{H} \beta$ component with an upper limit in the FWHM of $1200 \mathrm{~km} \mathrm{~s}^{-1}$. The [O III] $\lambda \lambda 4959$, 5007 doublets were modeled using two Gaussian functions, where an upper limit of the FWHM of the core component was set to $1200 \mathrm{~km} \mathrm{~s}^{-1}$. During the fit, the flux ratio of [O III] $\lambda 4959$ and [O III] $\lambda 5007$ was fixed to its theoretical value. The spectral decomposition was applied to each nightly spectrum; an example of such a decomposition is shown in Fig. 1.

To minimize any systematic uncertainty due to the decompositions of broad and narrow $\mathrm{H} \beta$ components, the total (broad + narrow) $\mathrm{H} \beta$ best-fit model was used to estimate $\mathrm{H} \beta$ line flux. The $\mathrm{H} \gamma$ emission line complex consists of broad and narrow $\mathrm{H} \gamma$ and [O III] $\lambda 4363$ lines. Since $\mathrm{H} \gamma$ is much weaker than $\mathrm{H} \beta$, the spectral decomposition is difficult to perform, especially for low $\mathrm{S} / \mathrm{N}$ spectra. Therefore, instead of modeling the $\mathrm{H} \gamma$ complex, emission line flux was directly integrated using the best-fit continuum (AGN power-law and Fe II) subtracted spectra. Furthermore, due to low $\mathrm{S} / \mathrm{N}$ in some epochs and blending with [O III] 14959, $\mathrm{H} \beta$ line wings are not well constrained. Therefore, to minimize any systematic uncertainty in spectral decomposition, the $\mathrm{H} \beta$ and $\mathrm{H} \gamma$ line fluxes were integrated within 4800-4930 and 4290-4410 $\AA$, respectively, to avoid the line wings.

Uncertainties in the spectral model parameters (e.g., flux, FWHM, $\alpha$ ) were estimated using a Monte Carlo simulation. For each observed spectrum, 100 mock spectra were generated, adding Gaussian random deviates of zero mean and the associated observed flux uncertainty of sigma. Then the same spectral decomposition method was repeated on the mock spectra,

\footnotetext{
2 http://purl.com/net/mpfit
} 


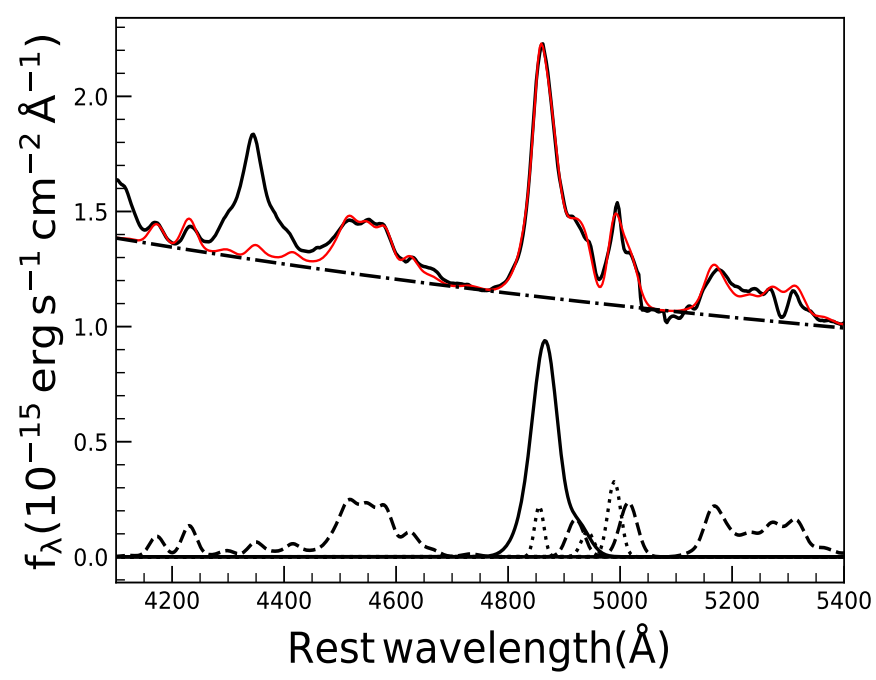

Fig. 1. Example of the spectral decomposition of PKS 1510-089. The rest-frame spectrum (black), best-fit model (red), and decomposed AGN power-law component (dashed-dot) are shown along with the Fe II emission (dashed), broad $\mathrm{H} \beta$ (solid), and narrow $\mathrm{H} \beta$ and [O III] (dotted).

as was done for the observed spectrum. The distribution of each parameter from the 100 mock spectra for each original spectrum allowed me to calculate $1 \sigma(68 \%)$ dispersion, which I considered the measurement uncertainty of that parameter.

The final analysis was performed on 271 spectra; 70 spectra with poor continuum fitting and low $\mathrm{S} / \mathrm{N}$ were excluded. The final $5100 \AA, \mathrm{H} \beta$, and $\mathrm{H} \gamma$ spectroscopic light curves are shown in Fig. 2 and given in Table 1 . The variation of the optical spectral index with time is shown. The $\gamma$-ray and radio $15 \mathrm{GHz}$ light curves are also shown in Fig. 2.

\subsection{Variability}

In order to characterize the flux variation in different wavelengths, the fractional root-mean-square (rms) variability amplitude was calculated following Rodríguez-Pascual et al. (1997):

$F_{\mathrm{var}}=\frac{\sqrt{\sigma^{2}-\left\langle\delta^{2}\right\rangle}}{\langle f\rangle}$,

where $\sigma^{2}$ is the variance, $\left\langle\delta^{2}\right\rangle$ is the mean square error, and $\langle f\rangle$ is the arithmetic mean of the light curves. The ratio of maximum to minimum flux variation $\left(R_{\max }\right)$ was also calculated for photometric and spectroscopic light curves. The values are given in Table 2. The source shows strong variations in all bands from $\gamma$ ray to radio. Optical photometry and $5100 \AA$ spectroscopic light curves also show strong variation. Noteworthy is the correlation of flux variation in optical and $\gamma$-ray bands. There are two strong peaks at Modified Julian Date (MJD) $=54900$ and 57 150, where the optical flux shows correlated variation with $\gamma$ rays; however, emission lines do not show any correlated peaks, suggesting that PKS 1510-089 has a significant non-thermal synchrotron contribution.

To determine if the continuum variability of PKS 1510-089 is dominated by the accretion disk (thermal contribution) or the jet (non-thermal synchrotron contribution), the non-thermal dominance (NTD) parameter (Shaw et al. 2012) was calculated following Patino-Alvarez et al. (2016):

$\mathrm{NTD}=\frac{L_{\mathrm{o}}}{L_{\mathrm{p}}}=\frac{\left(L_{\mathrm{d}}+L_{\mathrm{j}}\right)}{L_{\mathrm{p}}}$, where $L_{\mathrm{o}}$ and $L_{\mathrm{p}}$ are the observed continuum luminosity and predicted disk continuum luminosity estimated from the broad emission line, respectively. The observed continuum luminosity of radio-loud sources is a combination of luminosity emitted from the accretion disk $\left(L_{\mathrm{d}}\right)$ and the jet $\left(L_{\mathrm{j}}\right)$. Therefore, if the thermal emission from the disk is only responsible for ionizing the broad line clouds, then $L_{\mathrm{p}}=L_{\mathrm{d}}$ and NTD $=1+L_{\mathrm{j}} / L_{\mathrm{d}}$. If the continuum is only due to the thermal contribution from the disk, then NTD $=1$; however, if the jet also contributes to the continuum luminosity, then NTD $>1$. In the case of jet contribution greater than disk contribution, NTD can be larger than 2 . To estimate $L_{\mathrm{p}}$, the correlation of $L(\mathrm{H} \beta)-L_{5100}$ (orthogonal least square) obtained by Rakshit et al. (2020) for non-blazars SDSS DR14 quasars and the $L(\mathrm{H} \beta)$ estimated in this work were used. The variation of NTD with time is shown in the last panel of Fig. 2. I note the following points: (1) The NTD varies between 1 and 2 most of the time, suggesting that the non-thermal emission from the jet contributes to the continuum variation, and thermal disk contribution dominates over the jet contribution in the continuum variation. (2) At a few instants, MJD $=54900$ and 57150 , the NTD shows strong spikes, which are correlated with the flaring event in the $\gamma$-ray light curve, increasing up to 5 and 7 , respectively.

The correlation between the continuum and emission line luminosity of PKS 1510 was studied. In Fig. 3, H $\beta$ luminosity (upper panel) and NTD (bottom panel) are plotted against $5100 \AA$ continuum luminosity. The NTD gradually increases from 1 to 7 with $L_{5100}$; however, it remains $<2$ until $\log L_{5100} \sim$ 45.6 and increases rapidly for $\log L_{5100}>45.6$, reaching NTD $\sim 7$ for the maximum luminosity of $\log L_{5100} \sim 46$. A positive (though weak) correlation between $L(\mathrm{H} \beta)$ and $L_{5100}$ is found, with a Spearman correlation coefficient $\left(r_{\mathrm{s}}\right)$ of 0.39 and a $p$-value of no-correlation of $10^{-11}$. This correlation becomes strong with $r_{\mathrm{s}}=0.71\left(p\right.$-value of $\left.10^{-35}\right)$, when sources with NTD $<2$ (dotted line) are considered.

The spectral slope $\alpha\left(f_{\lambda} \propto \lambda^{\alpha}\right)$ with $L_{5100}$ is plotted in the middle panel of Fig. 3. The value of $\alpha$ increases with luminosity. For high luminosity $\log L_{5100}>45.6, \alpha$ is saturated and no correlation is found with brightness. Those epochs have NTD $<2$. The median value of $\alpha$ is $-1.19 \pm 0.31$. A positive correlation in the $\alpha-\log L_{5100}$ relation is found with $r_{\mathrm{s}}=0.43$ and a $p$-value of $10^{-13}$. Therefore, a "redder when brighter" (RWB) trend is observed in PKS 1510, indicating the presence of an accretion disk in the continuum (e.g., Gu et al. 2006; Nalewajko et al. 2019).

\subsection{Time delay measurement}

As found in the previous section, the optical continuum light curve of PKS 1510-089 has a non-thermal contribution, which is dominant at some epochs where the $\gamma$-ray light curve shows flare. However, broad emission line clouds do not respond to this variation. Therefore, to estimate the time delay between the continuum and emission line variation, a part of the light curve between MJD = 55000-57 100 was used, denoted by the vertical line (hereafter "window A").

The spectroscopic light curve of PKS 1510 shows a longterm trend. Such trends, which are not due to the reverberation variation (see Welsh 1999), have been reported in previous RM studies (e.g., Denney et al. 2010; Zhang et al. 2019). Welsh (1999) suggested fitting a low-order (at least linear) polynomial to the light curve and subtracting it from the light curve (i.e., detrending) to improve the cross-correlation results. Therefore, 

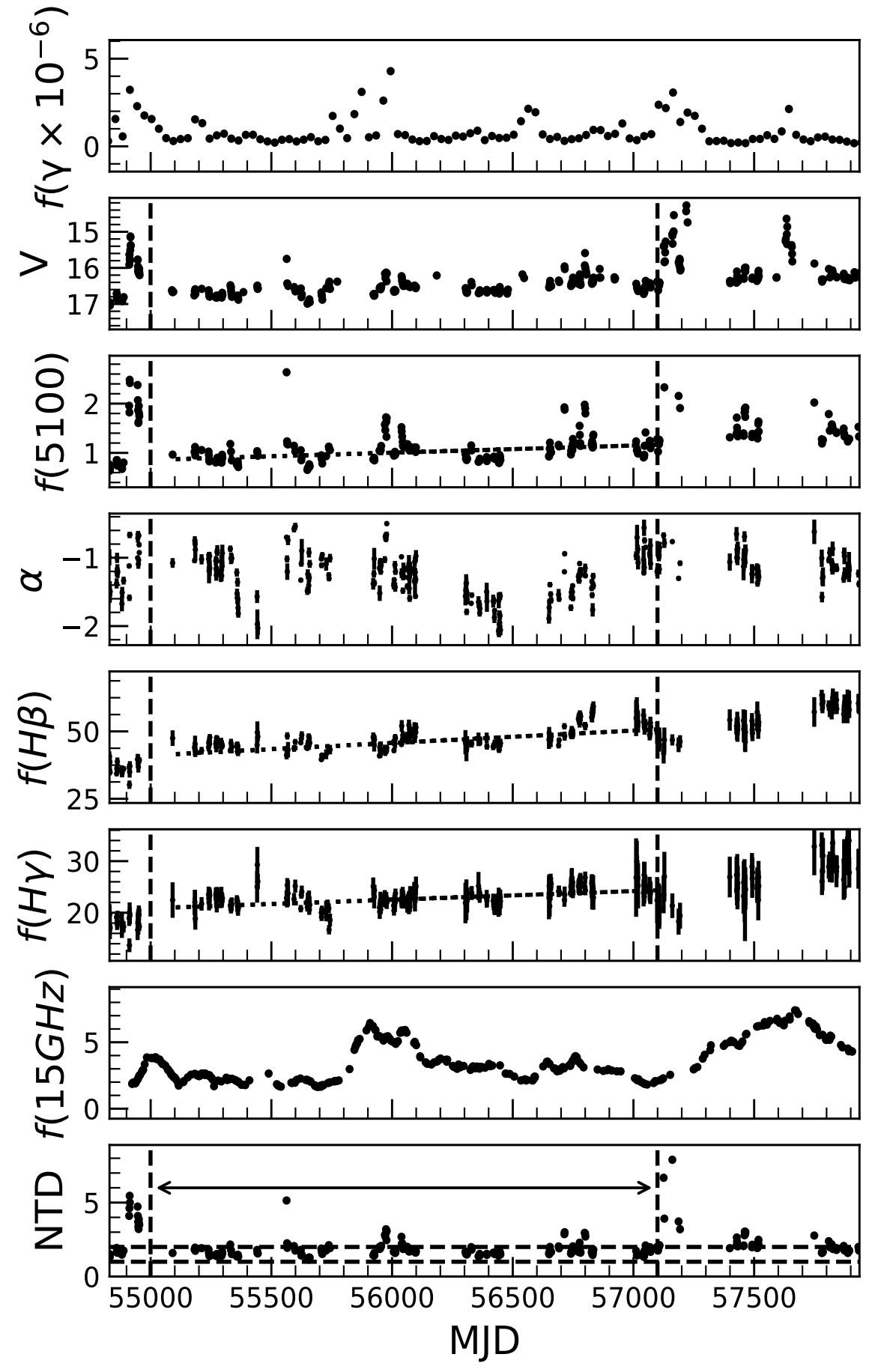

Fig. 2. Light curves of PKS 1510-089. From top to bottom: variation of $\gamma$ ray, $V$-band, $5100 \AA$ continuum, spectral index, $\mathrm{H} \beta, \mathrm{H} \gamma$, radio, and non-thermal dominance (NTD; see text) with times. The unit of $\gamma$-ray flux is photons $\mathrm{s}^{-1} \mathrm{~cm}^{-2}, f_{5100}$ is $10^{-15} \mathrm{erg} \mathrm{s}^{-1} \mathrm{~cm}^{-2} \AA^{-1}$, emission line flux is $10^{-15} \mathrm{erg} \mathrm{s}^{-1} \mathrm{~cm}^{-2}$, and radio flux is Jy. The section between MJD $=55000-57100$ (window A) of the spectroscopic light curves used in the time series analysis is represented by the vertical lines. The dotted line in the spectroscopic light curve is a linear fit to the data for detrending. Two horizontal lines at NTD $=1$ and 2 are shown in the lower panel.

Table 1. Spectroscopic data.

\begin{tabular}{lcccccc}
\hline \hline $\begin{array}{l}\text { MJD } \\
(1)\end{array}$ & $f_{5100}$ & $f(\mathrm{H} \beta)$ & $f(\mathrm{H} \gamma)$ & $f_{5100}^{*}$ & $f(\mathrm{H} \beta)^{*}$ & $f(\mathrm{H} \gamma)^{*}$ \\
\hline 54829 & $0.764 \pm 0.010$ & $40.785 \pm 1.821$ & $20.094 \pm 1.636$ & $1.123 \pm 0.010$ & $48.302 \pm 1.821$ & $20.094 \pm 1.636$ \\
54830 & $0.636 \pm 0.007$ & $35.713 \pm 2.058$ & $19.649 \pm 1.761$ & $0.995 \pm 0.007$ & $43.226 \pm 2.058$ & $19.649 \pm 1.761$ \\
54831 & $0.647 \pm 0.010$ & $38.696 \pm 2.291$ & $17.356 \pm 2.461$ & $1.006 \pm 0.010$ & $46.204 \pm 2.291$ & $17.356 \pm 2.461$ \\
\hline
\end{tabular}

Notes. Columns are: (1) modified Julian date (MJD); (2) monochromatic continuum flux at $5100 \AA$ in units of $10^{-15} \mathrm{erg} \mathrm{s}^{-1} \mathrm{~cm}^{-2} \AA^{-1}$; (3) and (4) are the $\mathrm{H} \beta$ and $\mathrm{H} \gamma$ line fluxes, respectively, in units of $10^{-15} \mathrm{erg} \mathrm{s}^{-1} \mathrm{~cm}^{-2}$. The detrended light curves are marked with * symbol (Cols. 5-7). The table is available in its entirety in a machine-readable form at the CDS version. A portion is shown here for guidance regarding its form and content. 
Table 2. Variability statistics.

\begin{tabular}{lcccc}
\hline \hline Light curve & Median flux & $F_{\text {var }}(\%)$ & $R_{\max }$ & $\begin{array}{c}\text { Cadence } \\
\text { entire (season) } \\
(1)\end{array}$ \\
$(2)$ & $(3)$ & $(4)$ & $(5)$ \\
\hline$f_{5100}$ & $1.09 \pm 0.44$ & $37.30 \pm 0.06$ & $7.36 \pm 0.08$ & $11.4(6.3)$ \\
$\mathrm{H} \beta$ & $46.96 \pm 6.10$ & $11.88 \pm 0.29$ & $2.09 \pm 0.12$ & - \\
$\mathrm{H} \gamma$ & $22.92 \pm 3.18$ & $9.61 \pm 0.71$ & $2.51 \pm 0.58$ & - \\
\hline
\end{tabular}

Notes. Columns are: (1) light curve and (2) median flux of the light curve in units of $10^{-15} \mathrm{erg} \mathrm{s}^{-1} \mathrm{~cm}^{-2} \AA^{-1}$ for $f_{5100}$, and $10^{-15} \mathrm{erg} \mathrm{s}^{-1} \mathrm{~cm}^{-2}$ for emission lines; (3) fractional rms variability in percentage; (4) the ratio of maximum to minimum flux variation; and (5) average cadence in days over the entire light curve and over each season in parenthesis.
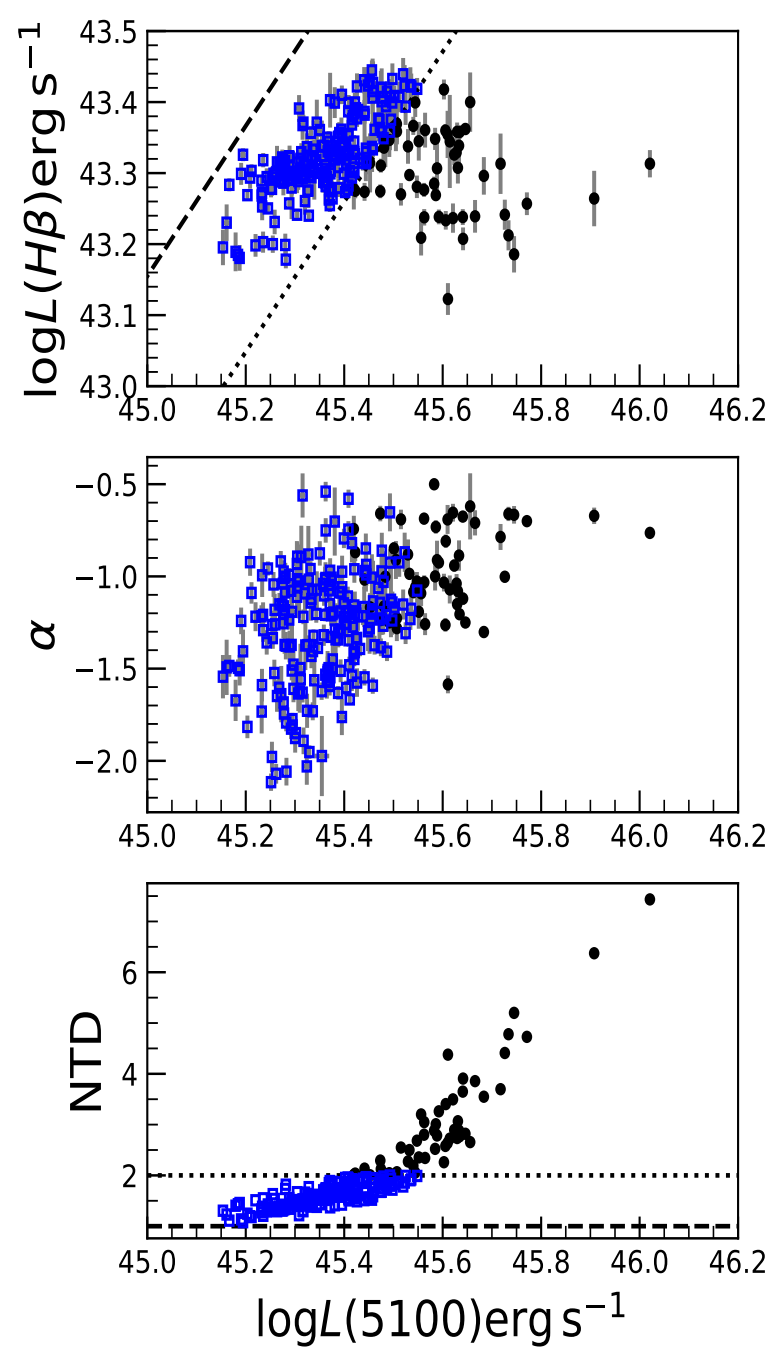

Fig. 3. Correlation of $\mathrm{H} \beta$ line luminosity (upper panel), optical spectral index (middle panel), and NTD (bottom panel) with $L_{5100}$ during the monitoring period. The empty squares are the epochs with NTD $<2$, while filled circles are those with NTD $\geq 2$. The dashed and dotted lines represent NTD $=1$ and 2 , respectively.

each spectroscopic light curve was detrended prior to the crosscorrelation analysis. The linear fits to the $f_{5100}, f(\mathrm{H} \beta)$, and $f(\mathrm{H} \gamma)$ light curves are indicated in Fig. 2 by the dashed line and the detrended spectroscopic light curves are shown in Fig. 4.
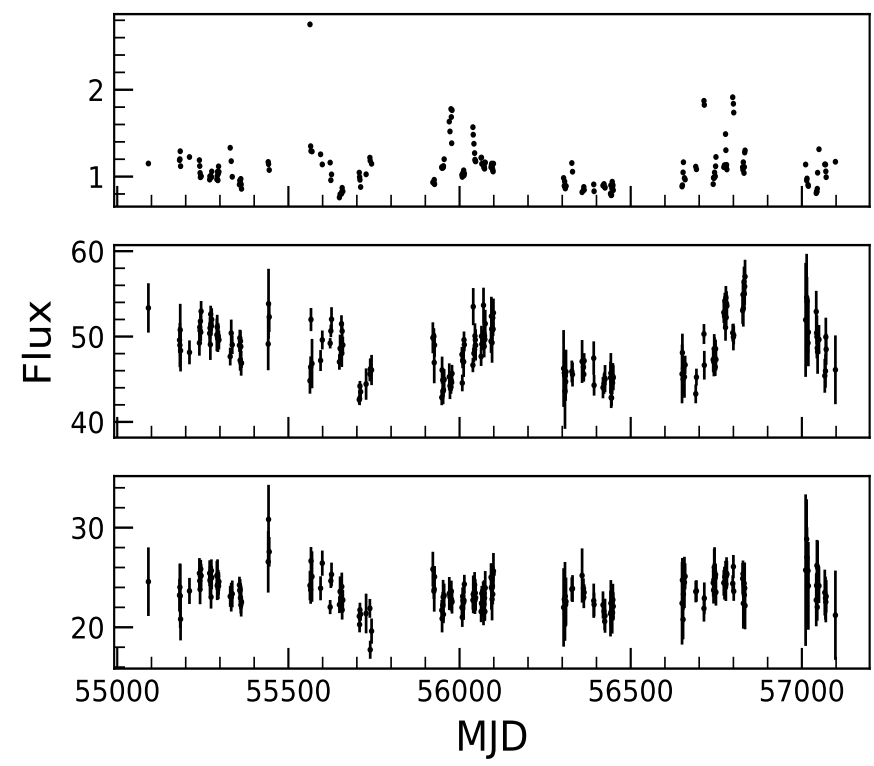

Fig. 4. Detrended spectroscopic light curves used in the time delay analysis. From top to bottom: $f_{5100}$ continuum and $\mathrm{H} \beta$ and $\mathrm{H} \gamma$ line light curves. The units are $10^{-15} \mathrm{erg} \mathrm{s}^{-1} \mathrm{~cm}^{-2} \AA^{-1}$ for $f_{5100}$ and $10^{-15} \mathrm{erg} \mathrm{s}^{-1} \mathrm{~cm}^{-2}$ for the line light curves. The point above $2 \times$ $10^{-15} \mathrm{erg} \mathrm{s}^{-1}$ in the $f_{5100}$ light curve is excluded from the time delay analysis.

\subsubsection{Cross-correlation analysis}

The cross-correlation technique (Gaskell \& Peterson 1987; White \& Peterson 1994; Peterson et al. 2004) was used to measure the time delay. The interpolated cross-correlation function (ICCF) was calculated following the description in Peterson et al. (2004). First, the cross-correlation function (CCF) was calculated with the interpolated continuum light curve while keeping the line light curve unchanged. Then the CCF was recalculated with the interpolated line light curve while keeping the continuum light curve unchanged. The average of the two CCFs provided the final ICCF. Additionally, the discrete correlation function (DCF) was measured following Edelson \& Krolik (1988). The centroid of the CCF ( $\left.\tau_{\text {cent }}\right)$ was calculated using the points at $80 \%$ of the CCF peak.

To estimate the uncertainty in $\tau_{\text {cent }}$, the flux randomization and random subset sampling (FR-RSS) method was used (Peterson et al. 1998, 2004). This was done using Monte Carlo realizations of the light curves. First, a mock light curve was created, adding Gaussian noise based on the associated flux uncertainty. Second, the same number of points as in the original light curve were randomly selected; if one epoch was selected $n$ times, the uncertainty of the flux was reduced by $n^{1 / 2}$. A total of 5000 mock light curves were generated and $\tau_{\text {cent }}$ was estimated the same way as for the original light curve. The median of the $\tau_{\text {cent }}$ distribution was taken as the final $\tau_{\text {cent }}$ and its upper and lower uncertainties were calculated such that $15.87 \%$ of the realizations fall above the range of uncertainties and $15.87 \%$ fall below.

The ICCF and DCF between $f_{5100}$ and the $\mathrm{H} \beta$ and $\mathrm{H} \gamma$ emission line light curves are shown in Fig. 5, both before and after detrending. In Table 3, the results of the cross-correlation analysis are given. Both the ICCF and DCF methods show consistent results. First, an RM lag is clearly seen from the crosscorrelation analysis for the $\mathrm{H} \beta$ and $\mathrm{H} \gamma$ lines both before and after detrending, as the highest significant peak is at the same position and remains unchanged. Second, for both the $\mathrm{H} \beta$ and $\mathrm{H} \gamma$ light 

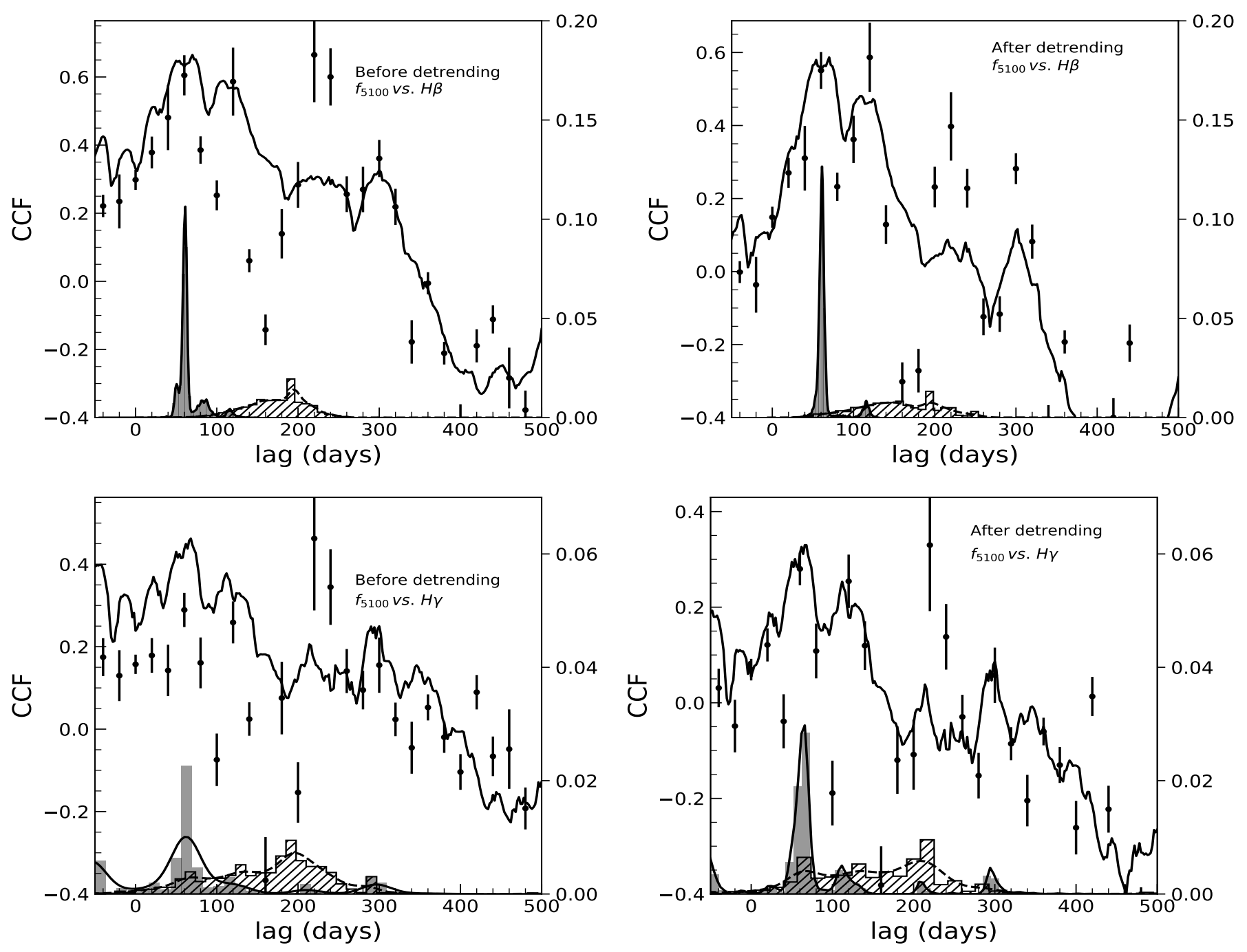

Fig. 5. Cross-correlation analysis of $f_{5100}$ vs. $\mathrm{H} \beta$ (top panels) and $\mathrm{H} \gamma$ (bottom panels) light curves before (left-hand panel) and after detrending (right-hand panel). The ICCF (line) and DCF (points) are shown. The centroid probability distribution from ICCF (filled histogram) and DCF (hatched histogram), along with smooth kernel density (solid and dashed lines, respectively), are also shown.

curves, the peak of the CCF before detrending is much broader or flatter than that after detrending, although the maximum correlation coefficient $\left(r_{\max }\right)$ is slightly higher in the former. Since the CCF after detrending is much narrower and the estimated lags are well constrained, the detrend lag measurement was therefore adopted for further analysis.

\subsubsection{The von Neumann and Bartels estimators}

Chelouche et al. (2017) introduced a method to measure time lag based on the regularity or randomness of data. This method requires neither interpolation or binning, nor the stochastic modeling of the light curves. They found that the von Neumann mean-square successive-difference estimator (von Neumann 1941) provides better time delay measurements for irregularly sampled time series where the underlying variability process can not be modeled properly. A detailed description of this method is given in Chelouche et al. (2017). To estimate the time delay between detrended $f_{5100}$ and the line light curves of PKS 1510, a publicly available python $\operatorname{code}^{3}$ for an optimized von Neumann

\footnotetext{
3 http://www.pozonunez.de/astro_codes/python/vnrm.py
}

estimator is used. The time delay distribution obtained from the von Neumann method after a Monte Carlo simulation of FRRSS, as done for the CCF analysis, is shown in the upper panels of Fig. 6. Both $\mathrm{H} \beta$ and $\mathrm{H} \gamma$ show strong peaks at $\sim 60$ days; however, two additional peaks at around 200 and 300 days are also present. A modification of the von Neumann estimator is the Bartels estimator (Bartels 1982), which can also be used to measure time delay based on the regularity or randomness of data. The time delay distribution based on the Bartels estimator is shown in the lower panels of Fig. 6. Unlike the von Neumann estimator, the Bartels estimator shows a single prominent peak in the distribution. The peaks at around 200 days are absent for both the $\mathrm{H} \beta$ and $\mathrm{H} \gamma$ light curves, and the peaks at 300 days are insignificant compared to the prominent peak at around 60 days. Lag results are given in Table 3.

\subsubsection{JAVELIN}

Time delay was also measured by modeling the continuum and line light curves using the JAVELIN code developed by $\mathrm{Zu}$ et al. (2011, 2013). JAVELIN first models the driving continuum light curve using a damped random walk (DRW) process (e.g., 
Table 3. Time delay analysis results.

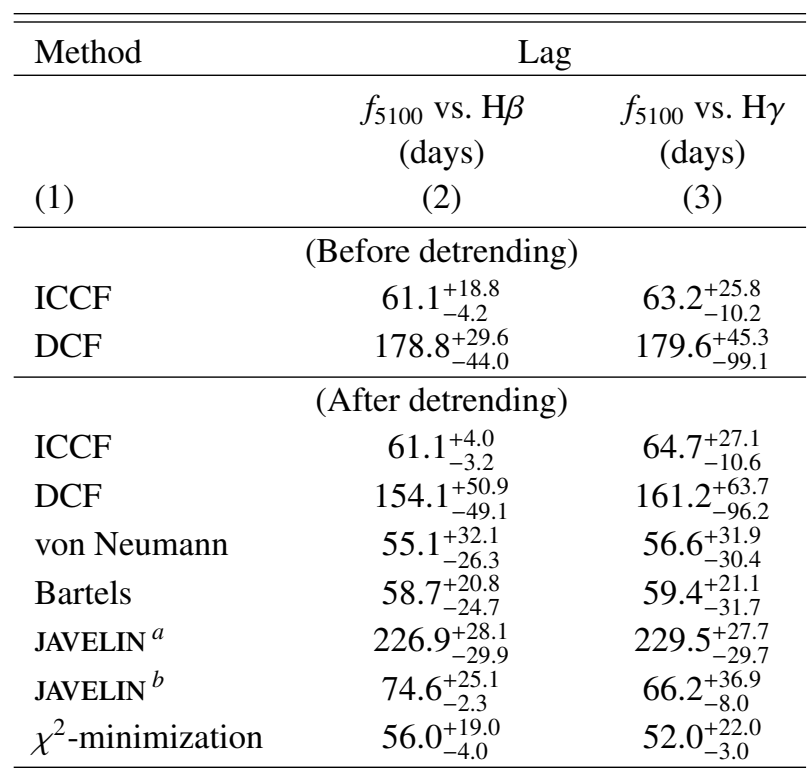

Notes. Columns are as follows: (1) method used; (2) and (3) lags for $f_{5100}$ vs. $\mathrm{H} \beta$ and $f_{5100}$ vs. $\mathrm{H} \gamma$ light curves. All the lags are in the observed frame. ${ }^{(a)}$ Lag search is allowed between 0 and 500 days. ${ }^{(b)}$ Lag search is allowed between 0 and 180 days.

Kelly et al. 2009) with two parameters: amplitude and time scale of variability. The emission line light curve is a shifted, scaled, and smoothed version of the continuum light curve. JAVELIN then uses a Markov chain Monte Carlo (MCMC) approach to maximize the likelihood of simultaneously modeling the continuum and line light curves. In Fig. 7, the probability distribution of the observed frame lag is plotted in the left-hand panel, as computed by JAVELIN when a lag search is allowed between 0 and 500 days (as was done for the CCF and von Neumann cases). JAVELIN shows prominent peaks at $\sim 200$ and $\sim 250$ days for both $\mathrm{H} \beta$ and $\mathrm{H} \gamma$. The peaks around $\sim 60$ days, which are found using the ICCF, von Neumann, and Bartels methods, are not visible. To find any peak at a lower lag, JAVELIN was allowed to search for lags between 0 and 180 days by refitting the light curves. The resultant lag probability distribution is shown in the right-hand panels of Fig. 7 . In this case, a prominent peak at $\sim 70$ days is found for both the $\mathrm{H} \beta$ and $\mathrm{H} \gamma$ light curves. Time delays obtained from JAVELIN are given in Table 3.

\subsubsection{The $\chi^{2}$-minimization method}

Czerny et al. (2013) find that $\chi^{2}$-minimization is a useful method to measure time lag. Therefore, to calculate time lag, $\chi^{2}$-minimization was also applied. First, the mean values were subtracted from the light curves and then normalized by their corresponding standard deviation. The continuum light curve was then linearly interpolated to the emission line light curve and the degree of similarity was calculated by time-shifting the line light curve using the $\chi^{2}$-minimization method. The time lag at which $\chi^{2}$ shows the minimum was considered as the most likely time lag. The final time lag and its uncertainty were calculated using the FR-RSS method, as done in the CCF analysis, for 5000 iterations. The lag probability distribution obtained from $\chi^{2}$-minimization is shown in Fig. 8 and lag values are given in Table 3. The distribution shows a strong peak at $\sim 60$ days for
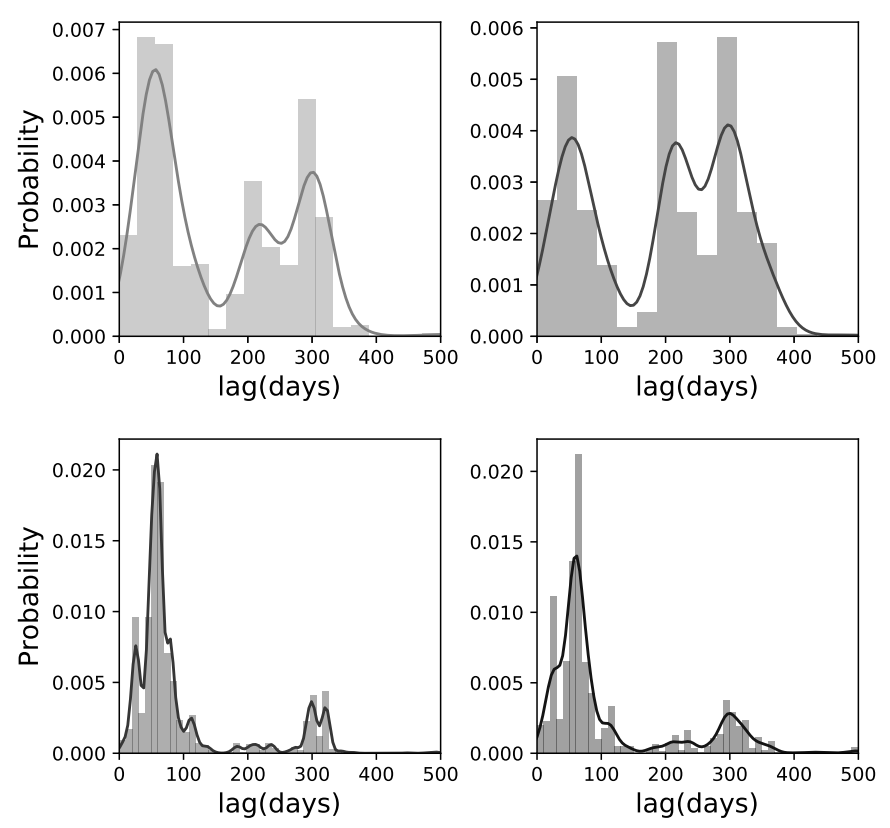

Fig. 6. Probability distribution of the observed frame time lag based on the von Neumann estimator (top panels) and the Bartels estimator (bottom panels) for $\mathrm{H} \beta$ (left) and $\mathrm{H} \gamma$ (right).
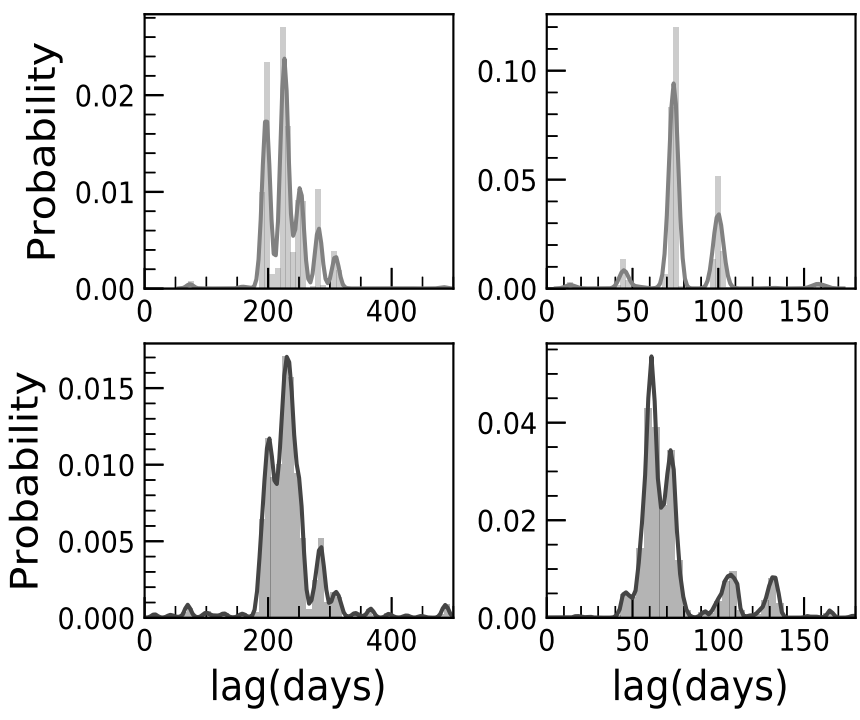

Fig. 7. Probability distribution of observed frame lag computed by JAVELIN when lag search is allowed between 0 and 500 (left-hand panels) and 0-180 days (right-hand panels) for $\mathrm{H} \beta$ (upper panels) and $\mathrm{H} \gamma$ (lower panels).

both $\mathrm{H} \beta$ and $\mathrm{H} \gamma$. Although a small peak at $\sim 300$ days can be found for $\mathrm{H} \gamma$, no such peak is found for $\mathrm{H} \beta$.

The above methods strongly suggest a time lag of $\sim 60$ days between the continuum and the $\mathrm{H} \beta$ light curve. To visually check the consistency of the measured lag, the $f_{5100}$ light curve along with the $\mathrm{H} \beta$ light curve back-shifted by 60 days are plotted in Fig. 9. The continuum and back-shifted line light curves match well. Therefore, I adopted the lag of $61.1_{-3.2}^{+4.0}$ days obtained by the ICCF method after detrending as the best lag measurement for PKS 1510. 

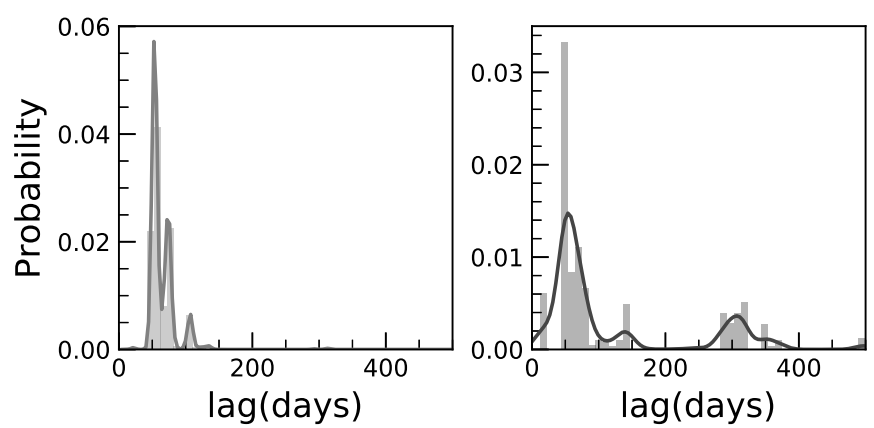

Fig. 8. Probability distribution of observed frame lag computed based on the $\chi^{2}$ minimization method.

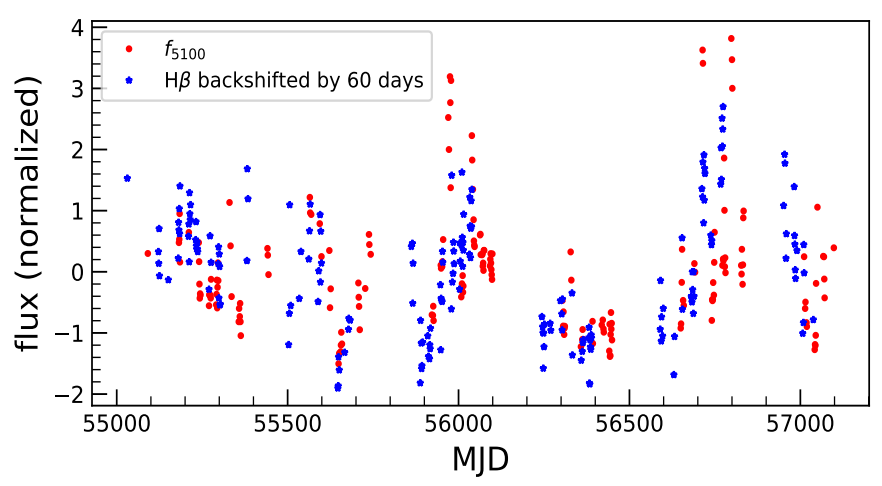

Fig. 9. Normalized $f_{5100}$ light curve plotted along with the $\mathrm{H} \beta$ light curve back-shifted by 60 days.

\subsection{Line width and black hole mass}

The mean and rms spectra were constructed from the nightly spectrum observed in window A following Rakshit et al. (2019). The mean spectrum is

$\langle f(\lambda)\rangle=\frac{1}{N} \sum_{i=1}^{N} f_{i}(\lambda)$.

Here, $f_{i}(\lambda)$ is the $i$ th spectrum. The rms spectrum is

$\Delta(\lambda)=\sqrt{\left[\frac{1}{N-1} \sum_{i=1}^{N}\left[f_{i}(\lambda)-\langle f(\lambda)\rangle\right]^{2}\right]}$,

where the integration runs from 1 to the total number of spectra $(N)$. The mean and rms spectra are shown in Fig. 10. The rms spectrum clearly shows variations in both the $\mathrm{H} \beta$ and $\mathrm{H} \gamma$ lines.

The FWHM and line dispersion (second moment, $\sigma_{\text {line }}$ ) were measured from the mean and rms spectra constructed from the nightly spectrum after subtracting the power-law and Fe II component. The FWHM was calculated using the methodology described in Peterson et al. (2004). To measure $\sigma_{\text {line }}$, the flux weighted line center was first determined as follows:

$\lambda_{0}=\frac{\int \lambda f_{\lambda} \mathrm{d} \lambda}{\int f_{\lambda} \mathrm{d} \lambda}$

and then the line dispersion as

$\sigma_{\text {line }}^{2}=\frac{\int \lambda^{2} f_{\lambda} \mathrm{d} \lambda}{\int f_{\lambda} \mathrm{d} \lambda}-\lambda^{2}{ }_{0}$,

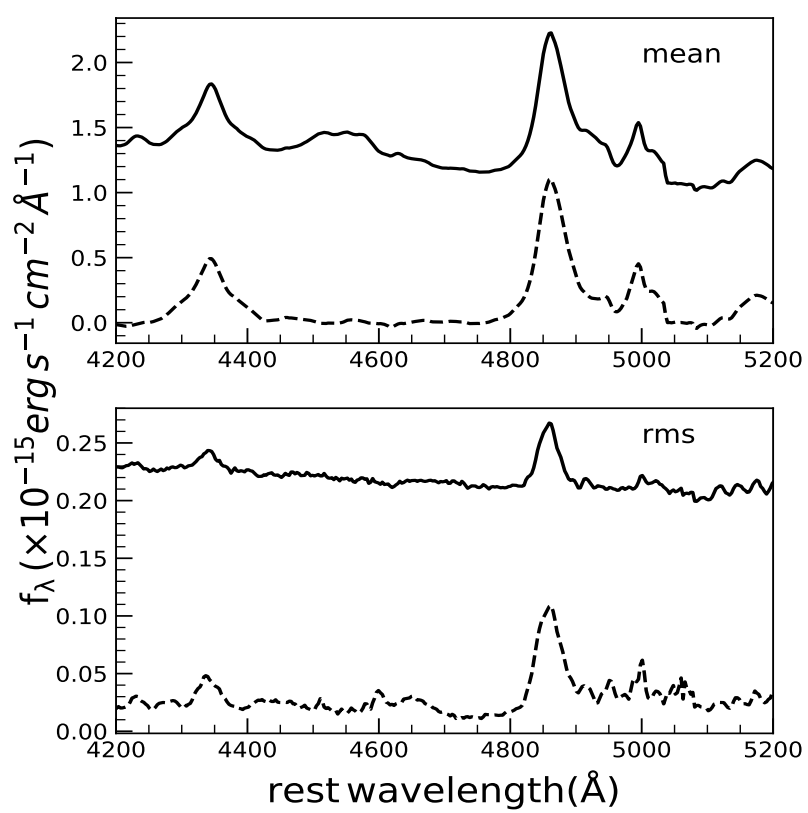

Fig. 10. Mean and rms spectra of PKS 1510-089. Top panel: standard mean spectrum (solid line) and a mean spectrum constructed after subtracting the power-law and Fe II (dashed line). Bottom: same as the top panel but for the rms spectrum.

Table 4. Rest-frame resolution-corrected line width and black hole mass measurements from mean and rms spectra created from the nightly spectrum after subtracting the power-law and Fe II component.

\begin{tabular}{lccc}
\hline \hline Spectrum & Type & $\begin{array}{c}\Delta V \\
\left(\mathrm{~km} \mathrm{~s}^{-1}\right)\end{array}$ & $\begin{array}{c}M_{\mathrm{BH}} \\
\left(\times 10^{7} M_{\odot}\right) \\
(1)\end{array}$ \\
\hline Mean & $F W H M$ & $2673 \pm 15$ & $7.02_{-0.36}^{+0.46}$ \\
& $\sigma_{\text {line }}$ & $1378 \pm 64$ & $7.45_{-0.52}^{+0.59}$ \\
rms & $F W H M$ & $2066 \pm 76$ & $4.19_{-0.26}^{+0.31}$ \\
& $\sigma_{\text {line }}$ & $1207 \pm 105$ & $5.71_{-0.58}^{+0.62}$ \\
\hline
\end{tabular}

Notes. Columns are: (1) spectrum type; (2) line width indicator; (3) line width; and (4) black hole mass.

where $f_{\lambda}$ is the mean or the rms spectra. The endpoints of the integrations were selected visually to be $4800-4930 \AA$ for $\mathrm{H} \beta$. To estimate uncertainty in the line width measurements, the Monte Carlo bootstrap method (Peterson et al. 2004) was used. For each realization, $N$ spectra were randomly selected from a set of $N$ spectra without replacement and the line width was calculated from the mean and rms spectra. In each realization, the endpoints of the integration window were randomly varied within $\pm 10 \AA$ from the initial selections. A total of 5000 realizations were performed, providing a distribution of FWHM and $\sigma_{\text {line. }}$. The median of the distribution was taken as the final line width and the standard deviation of the distribution was considered to be the measurement uncertainty. The final line width measurements, after correcting for the instrumental resolution of FWHM $\sim 1150 \mathrm{~km} \mathrm{~s}^{-1}$ (see Zhang et al. 2019), are given in Table 4.

The black hole mass of PKS 1510-089 was determined using the virial relation as follows:

$M_{\mathrm{BH}}=f \frac{R_{\mathrm{BLR}} \Delta V^{2}}{G}$. 


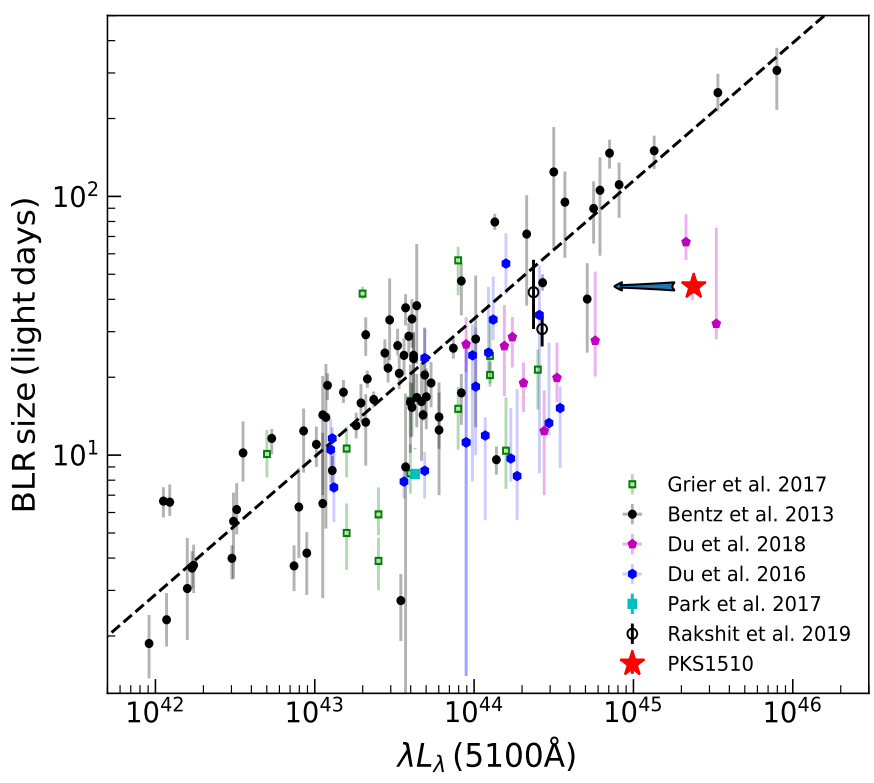

Fig. 11. PKS $1510-089$ in the BLR size vs. $L_{5100}$ relation of AGNs. The best-fit relation of Bentz et al. (2013) is shown along with various RM results from the literature. The arrow indicates that the accretion disk contribution to the measured $L_{5100}$ of PKS 1510-089 could be much lower.

Here, $f$ is the virial factor, $R_{\mathrm{BLR}}=c \tau$ is the BLR size in the rest-frame, and $\Delta V$ is the velocity width of the broad emission line.

The black hole mass was determined using an $\mathrm{H} \beta$ lag of $\tau_{\text {cent }}=61.1_{-3.2}^{+4.0}$ days, which corresponds to a rest-frame BLR size of $R_{\mathrm{BLR}}=c \tau_{\text {cent }} /(1+z)=44.9_{-2.3}^{+2.9}$ light-days. Both the resolution-corrected FWHM and $\sigma_{\text {line }}$ measured from the mean and $\mathrm{rms}$ spectra of $\mathrm{H} \beta$ were used as $\Delta V$. A value of $f=4.47$ (1.12) was adopted when $\sigma_{\text {line }}(\mathrm{FWHM})$ is considered as $\Delta V$ (Woo et al. 2015); however, I note that FWHM is a non-linear function of $\sigma_{\text {line }}$ (e.g., Peterson 2014; Bonta et al. 2020). Finally, four different black hole masses were determined based on the four different choices of line widths (see Table 4). The black hole masses and their uncertainties are calculated based on the error propagation method and given in Table 4 . I note that $\sigma_{\text {line }}$ is less sensitive to the line peak, and that FWHM is less sensitive to the line wing; therefore, black hole masses based on the $\sigma_{\text {line }}$ are widely adopted as the best mass measurement (e.g., Peterson et al. 2004; Peterson 2014). Therefore, $M_{\mathrm{BH}}=5.71_{-0.58}^{+0.62} \times 10^{7} M_{\odot}$ of PKS 1510-089 from $\sigma_{\text {line }}$ of rms spectrum was adopted in this work.

\section{Discussion}

\subsection{Impact of seasonal gaps on lag measurement}

The monitoring of PKS 1510 is strongly affected by seasonal gaps of about six months, which are due to its low declination. Therefore, any lag close to the average seasonal gaps of $\sim 180$ days will be difficult to measure because the emission-line response to the continuum variations occurs when the source is unobservable. The measured monochromatic luminosity of PKS 1510-089 from the mean spectra is $L_{5100}=2.39 \times 10^{45} \mathrm{ers} \mathrm{s}^{-1}$. Using the $R_{\mathrm{BLR}}-L_{5100}$ relation of a radio-quiet AGN sample presented by Bentz et al. (2013), an expected BLR size of $\sim 182$ days and an observed frame lag of 248 days are obtained for PKS
1510. As the expected lag is closer to the seasonal gaps, the impact of seasonal gaps on the lag measurement was investigated via the construction of mock light curves. For this purpose, a mock continuum light curve was constructed using the DRW model implemented in JAVELIN, which has a similar characteristic as the observed continuum light curve. Then mock line light curves with time delays of 70 and 200 days were constructed. To mimic the observed light curves, mock light curves were downsampled to have the same time sampling, and therefore the same time axis, as the observed light curves. To recover the input time delay, time series analysis methods, as described in Sect. 3.3, were used on the mock data sets.

The results are shown in Fig. A.1 and are given in Table A.1 of the appendix. The results show that the ICCF, DCF, von Neumann, and Bartels methods recover an input lag of 70 days, and the measurements are not affected by the seasonal gaps and time sampling. I note that the $r_{\max }$ obtained by ICCF and DCF is $\sim 0.6$, which is similar to that obtained in the case of the observed light curve (see Fig. 5). Interestingly, JAVELIN results are affected by seasonal gaps, as they show a primary peak at $\sim 160$ days. Due to the same reason, JAVELIN finds a lag at 200 days for the observed light curve of PKS 1510 when the lag search is allowed between 0 and 500 days (see Sect. 3.3.3). A secondary peak at $\sim 70$ days, which is the same as the input lag, is also found in the JAVELIN lag probability distribution, as shown in Fig. A.1. For an input lag of 200 days, both the ICCF and DCF show no correlation between the mock continuum and the line light curve due to the seasonal gaps; therefore, they do not allow me to estimate the lag. However, the von Neumann, Bartels, and JAVELIN methods successfully recover the input time lag of 200 days, albeit with larger uncertainty. Therefore, the uses of various time series analysis methods allow me to recover a lag of 200 days, although the light curves are affected by the seasonal gaps and a lag of $\sim 70$ days can be well-constrained with lower uncertainty. The above simulations suggest any lag close to 200 days is unlikely for PKS 1510 , while a lag of $\sim 60$ days is most likely.

\subsection{Size-luminosity relation}

The quasar PKS 1510-089 is a radio-loud source with strong $\gamma$-ray activity. From Fig. 3 , it is clear that the measured $L_{5100}$ is a combination of non-thermal synchrotron emission from the jet and thermal emission from the accretion disk. Hence, $L_{5100}$ measurement is strongly affected by the non-thermal emission. In Fig. 11, the BLR size of PKS $1510-089$ is plotted against $L_{5100}$, along with the previous reverberation mapped objects from the literature (e.g., Bentz et al. 2013; Du et al. 2016b; Grier et al. 2017; De Rosa et al. 2018; Rakshit et al. 2019); PKS 1510-089 is found to deviate from the $R_{\mathrm{BLR}}-L_{5100}$ relation of Bentz et al. (2013). However, this is not surprising considering the fact that previous studies of high-accreting and strong Fe II-emitting AGNs show significant deviation from the size-luminosity relation (Du et al. 2016a, 2018). This could be due to the complex radiation field and BLR geometry in high-accreting AGNs, which may have slim accretion disks. The strong self-shadowing effects on the slim accretion disks may produce a highly anisotropic radiation field, which, depending on the accretion rate, may lead to two dynamically distinct regions of the BLR (Wang et al. 2014). Du \& Wang (2019) find that the $R_{\mathrm{FeII}}$, (i.e., the flux ratio of Fe II to $\mathrm{H} \beta$ ) is the main driver of the shortened lag obtained in the high-accreting AGNs. They provide a new scaling relation, which includes $R_{\text {FeII }}$. Using the mean $R_{\text {FeII }}$ of $0.52 \pm 0.09$ found in PKS 1510-089, the expected lag is about $\sim 122$ days, which is lower than what is expected from 
the Bentz et al. (2013) relation but still a factor of three larger than the measured lag. Several authors (Celotti et al. 1997; Abdo et al. 2010a; Nalewajko et al. 2012) have estimated the disk bolometric luminosity $\left(L_{\text {disk }}\right)$ of PKS $1510-089$, which is in the range of $3-7 \times 10^{45} \mathrm{erg} \mathrm{s}^{-1}$. This, based on a simple scaling relation of $R_{\mathrm{BLR}}=10^{17} \sqrt{L_{\mathrm{disk}} / 10^{45}} \mathrm{~cm}$ (Ghisellini \& Tavecchio 2009), provides $R_{\mathrm{BLR}}=66-102$ light-days. Our estimated rest-frame $R_{\mathrm{BLR}}$ is slightly lower than the above values.

Li et al. (2020) studied a well-known FSRQ, 3C273, and find that the optical continuum has two components of emissions, one from the accretion disk and another from the jet. The jet contribution is found to be $10-40 \%$ of the total optical emissions. Whiting et al. (2001) showed that the synchrotron radiation from the jet contributes to the optical band, thereby increasing the total optical continuum flux. However, due to beaming, this synchrotron component does not contribute to ionizing the emission line clouds. Figure 2 shows strong $\gamma$-ray activity in PKS 1510089 at MJD $\simeq 54900$ and 57150 . Although the light curve when PKS 1510-089 is mostly in a quiescent state is analyzed in this work, a non-thermal contribution from the jet is always present. To have a rough estimation of thermal contribution in $L_{5100}$, the median of the NTD was calculated in the quiescent state (window A). It is found to be $1.67_{-0.23}^{+0.31}$, indicating that the disk contribution to the measured $L_{5100}$ is about $60 \%$.

This contribution can also be roughly estimated from the broad-band SED. Prince et al. (2019) studied the broad-band SED of PKS 1510-089 in several active and quiescent states in 2015 (MJD = 57 000-57 350). They modeled the broad-band SED using a time-dependent two-zone emission model and estimated the synchrotron, synchrotron self-Compton (SSC), and inverse-Compton emission. They estimated the disk contribution at $5100 \AA$ to the total flux during one quiescent state (Q2 between MJD = 57 180-57 208; see Fig. 3 of Prince et al. 2019). The disk contribution is found to be $\sim 44 \%$ of the total flux at $5100 \AA$, lower than that estimated from the NTD but consistent within the margin of error. If the measured $L_{5100}$ is corrected assuming the disk contribution is $60 \%$ (based on the NTD calculation), the corrected $L_{5100}$ is $1.43 \times 10^{45} \mathrm{ers} \mathrm{s}^{-1}$. In fact, using the mean $\mathrm{H} \beta$ line luminosity of $2.04 \times 10^{43} \mathrm{erg} \mathrm{s}^{-1}$ and the scaling relation of $L(\mathrm{H} \beta)-L(5100)$ for radio quiet AGNs from Liu et al. (2006), $L_{5100}$ is found to be $1.40 \times 10^{45} \mathrm{ers} \mathrm{s}^{-1}$. Therefore, the measured $L_{5100}$ of PKS $1510-089$ is an upper-limit, as it is significantly affected by the non-thermal contribution from the jet. Furthermore, the host galaxy also contributes to the measured $L_{5100}$. Therefore, the measured $L_{5100}$ is an upper limit and the actual position of PKS 1510-089 in the size-luminosity diagram is highly uncertain.

\subsection{Black hole mass measurement}

Since PKS 1510-089 is a well-studied object, several authors have reported its black hole mass based on single-epoch spectrum, variability time scale, accretion disk modeling, etc. Here, the mass measurement is compared with those in the literature. The estimated black hole of PKS 1510-089 ranges from 4.19-7.02 $\times 10^{7} M_{\odot}$, depending on the choice of the line width and line profile used. The bolometric luminosity is found to be $21.51 \times 10^{45} \mathrm{erg} \mathrm{s}^{-1}$, based on the measured $L_{5100}$ from the mean spectrum using $L_{\mathrm{BOL}}=9 \times L_{5100}$ (Kaspi et al. 2000). The Eddington ratio $\left(\lambda_{\mathrm{EDD}}\right)$ is 2.98 , estimated using $L_{\mathrm{EDD}}=$ $1.26 \times 10^{38} M_{\mathrm{BH}}$ and the black hole mass based on the $\sigma_{\text {line }}$ of rms spectrum. However, using the corrected $L_{5100}$ due to jet contribution, $\lambda_{\mathrm{EDD}}=1.78$ is found, suggesting that PKS 1510-089 is accreting at a mildly super-Eddington rate. This value is similar to the $\lambda_{\mathrm{EDD}}=2.4$ found for another FSRQ, 3C273 (Zhang et al. 2019).

Oshlack et al. (2002), using single-epoch spectrum, estimated the black hole mass of PKS $1510-089$ to be $3.86 \times 10^{8} M_{\odot}$. Similarly, Xie et al. (2005), based on the minimum time scale of variability and single-epoch spectrum, estimated black hole masses of $M_{\mathrm{BH}}=1.1 \times 10^{8} M_{\odot}$ and $1.6 \times 10^{8} M_{\odot}$, respectively (see also Liu et al. 2006; Park \& Trippe 2017). I note that single-epoch black hole masses depend on the choice of the scaling relation, which shows significant scatter; highly accreting sources especially show larger offset from the $R_{\mathrm{BLR}}-L_{5100}$ relation. The choice of the line width measurement, FWHM or $\sigma_{\text {line }}$, also affects the single-epoch mass measurement. Reverberation mapping studies of AGNs, in which multiple emission lines have been observed, suggest $\sigma_{\text {line }}$ is a better measure for black hole mass than FWHM (Peterson et al. 2004). Moreover, an rms profile, which can only be constructed from multi-epoch spectra, should be used to estimate black hole masses, as it shows the variable component of the emission line; contaminating features - such as constant host-galaxy contribution and narrowline components, which are present in the single-epoch spectrum or in the mean spectrum - disappear in the rms spectrum (see Peterson 2014). Using UV data, Abdo et al. (2010a) estimated a black hole mass for PKS $1510-089$ as $M_{\mathrm{BH}}=5.4 \times 10^{8} M_{\odot}$. On the other hand, Castignani et al. (2017), using the Shakura \& Sunyaev (1973) model, estimated a black hole mass of $2.4 \times 10^{8} M_{\odot}$. Therefore, a large range of black hole masses, $1-9 \times 10^{8} M_{\odot}$, for PKS $1510-089$ has been reported in the literature. Considering the typical uncertainty of $\sim 0.5 \mathrm{dex}$ in the mass measurement (Shen 2013), the estimated black hole mass of PKS 1510-089 is smaller by a factor of 2-4 than the values reported in the literature.

\section{Conclusion}

The optical spectroscopic RM results of PKS 1510-089 from an $~ 8.5$-year long monitoring campaign carried out at Steward Observatory from December 2008 to June 2017 are presented. The nightly spectrum shows the presence of broad $\mathrm{H} \beta$, $\mathrm{H} \gamma$, and Fe II emission overlying on a blue continuum. During the monitoring program, both the optical continuum and $\mathrm{H} \beta$ line show strong variation with fractional rms variations $\left(F_{\text {var }}\right)$ of: $37.30 \pm 0.06 \%$ for $f_{5100} ; 11.88 \pm 0.29 \%$ for $\mathrm{H} \beta$; and $9.61 \pm 0.71 \%$ for $\mathrm{H} \gamma$ light curves. With the increase of $L_{5100}$ from $10^{45.2}$ to $10^{45.6} \mathrm{erg} \mathrm{s}^{-1}$, the $\mathrm{H} \beta$ line luminosity increases from $10^{43.2}$ to $10^{43.5} \mathrm{erg} \mathrm{s}^{-1}$ but decreases as $L_{5100}>10^{45.6} \mathrm{erg} \mathrm{s}^{-1}$. Although the optical continuum is dominated by thermal radiation from the accretion disk, non-thermal synchrotron contribution from the jet is clearly present, which does not contribute to ionizing the emission line clouds. From cross-correlation analysis, the $\mathrm{H} \beta$ and $\mathrm{H} \gamma$ lags are found to be $61.1_{-3.2}^{+4.0}$ days and $64.7_{-10.6}^{+27.1}$ days, respectively. This corresponds to a rest-frame BLR size of $44.9_{-2.3}^{+2.9}$ light-days for $\mathrm{H} \beta$ and $47.5_{-7.8}^{+19.9}$ light-days for $\mathrm{H} \gamma$. Using a scale factor of 4.47 and the $\sigma_{\text {line }}$ from the rms spectrum, which is constructed from the nightly spectrum after subtracting the power-law and Fe II, the black hole mass of PKS $1510-089$ is found to be $M_{\mathrm{BH}}=5.71_{-0.58}^{+0.62} \times 10^{7} M_{\odot}$.

Acknowledgements. Many thanks go to the referee for comments and suggestions that helped to improve the quality of the manuscript. Thanks to Prince Raj for providing their SED model component of PKS1510-089 and Neha Sharma for carefully reading the manuscript. This publication makes use of data products from the Fermi Gamma-ray Space Telescope and accessed from the 
Fermi Science Support Center. Data from the Steward Observatory spectropolarimetric monitoring project were used. This program is supported by Ferm Guest Investigator grants NNX08AW56G, NNX09AU10G, NNX12AO93G, and NNX15AU81G. This research has made use of data from the OVRO 40-m monitoring program (Richards et al. 2011) which is supported in part by NASA grants NNX08AW31G, NNX11A043G, and NNX14AQ89G and NSF grants AST-0808050 and AST-1109911

\section{References}

Abdo, A. A., Ackermann, M., Ajello, M., et al. 2009, ApJ, 700, 597 Abdo, A. A., Ackermann, M., Agudo, I., et al. 2010a, ApJ, 721, 1425 Abdo, A. A., Ackermann, M., Agudo, I., et al. 2010b, ApJ, 716, 30 Bach, U., Raiteri, C. M., Villata, M., et al. 2007, A\&A, 464, 175

Bartels, R. 1982, J. Am. Stat. Assoc., 77, 40

Beaklini, P. P. B., Dominici, T. P., \& Abraham, Z. 2017, A\&A, 606, A87

Bentz, M. C., Denney, K. D., Grier, C. J., et al. 2013, ApJ, 767, 149

Bentz, M. C., Peterson, B. M., Netzer, H., Pogge, R. W., \& Vestergaard, M. 2009, ApJ, 697, 160

Blandford, R. D., \& McKee, C. F. 1982, ApJ, 255, 419

Bonta, E. D., Peterson, B. M., Bentz, M. C., et al. 2020, ApJ, submitted [arXiv:2007.02963]

Cardelli, J. A., Clayton, G. C., \& Mathis, J. S. 1989, ApJ, 345, 245

Castignani, G., Pian, E., Belloni, T. M., et al. 2017, A\&A, 601, A30

Celotti, A., Padovani, P., \& Ghisellini, G. 1997, MNRAS, 286, 415

Chelouche, D., Pozo-Nuñez, F., \& Zucker, S. 2017, ApJ, 844, 146

Cho, H., Woo, J.-H., Hodges-Kluck, E., et al. 2020, ApJ, 892, 93

Czerny, B., Hryniewicz, K., Maity, I., et al. 2013, A\&A, 556, A97

De Rosa, G., Fausnaugh, M. M., Grier, C. J., et al. 2018, ApJ, 866, 133

Denney, K. D., Peterson, B. M., Pogge, R. W., et al. 2010, ApJ, 721, 715

Du, P., \& Wang, J.-M. 2019, ApJ, 886, 42

Du, P., Lu, K.-X., Zhang, Z.-X., et al. 2016a, ApJ, 825, 126

Du, P., Lu, K.-X., Hu, C., et al. 2016b, ApJ, 820, 27

Du, P., Zhang, Z.-X., Wang, K., et al. 2018, ApJ, 856, 6

Edelson, R. A., \& Krolik, J. H. 1988, ApJ, 333, 646

Gaskell, C. M., \& Peterson, B. M. 1987, ApJS, 65,

Ghisellini, G., \& Tavecchio, F. 2009, MNRAS, 397, 985

Grier, C. J., Trump, J. R., Shen, Y., et al. 2017, ApJ, 851, 21

Gu, M. F., Lee, C. U., Pak, S., Yim, H. S., \& Fletcher, A. B. 2006, A\&A, 450, 39

H.E.S.S. Collaboration (Abramowski, A., et al.) 2013, A\&A, 554, A107

Kaspi, S., Smith, P. S., Netzer, H., et al. 2000, ApJ, 533, 631

Kelly, B. C., Bechtold, J., \& Siemiginowska, A. 2009, ApJ, 698, 895

Kormendy, J., \& Ho, L. C. 2013, ARA\&A, 51, 511

Kovačević, J., Popović, L. Č., \& Dimitrijević, M. S. 2010, ApJS, 189, 15

Kushwaha, P., Chandra, S., Misra, R., et al. 2016, ApJ, 822, L13

Li, Y.-R., Zhang, Z.-X., Jin, C., et al. 2020, ApJ, 897, 18

Liu, Y., Jiang, D. R., \& Gu, M. F. 2006, ApJ, 637, 669

Malkan, M. A., \& Moore, R. L. 1986, ApJ, 300, 216
Markwardt, C. B. 2009, in Astronomical Data Analysis Software and Systems XVIII, eds. D. A. Bohlender, D. Durand, \& P. Dowler, ASP Conf. Ser., 411, 251

Marscher, A. P., Jorstad, S. G., Larionov, V. M., et al. 2010, ApJ, 710, L126

Nalewajko, K., Sikora, M., Madejski, G. M., et al. 2012, ApJ, 760, 69

Nalewajko, K., Gupta, A. C., Liao, M., et al. 2019, A\&A, 631, A4

Orienti, M., Koyama, S., D’ Ammando, F., et al. 2013, MNRAS, 428, 2418

Oshlack, A. Y. K. N., Webster, R. L., \& Whiting, M. T. 2002, ApJ, 576, 81

Park, J., \& Trippe, S. 2017, ApJ, 834, 157

Park, S., Woo, J.-H., Romero-Colmenero, E., et al. 2017a, ApJ, 847, 125

Park, D., Barth, A. J., Woo, J.-H., et al. 2017b, ApJ, 839, 93

Patino-Alvarez, V. M., Torrealba, J., Chavushyan, V., et al. 2016, Front. Astron. Space Sci., 3, 19

Peterson, B. M. 1993, PASP, 105, 247

Peterson, B. M. 2014, Space Sci. Rev., 183, 253

Peterson, B. M., Wanders, I., Horne, K., et al. 1998, PASP, 110, 660

Peterson, B. M., Ferrarese, L., Gilbert, K. M., et al. 2004, ApJ, 613, 682

Prince, R., Gupta, N., \& Nalewajko, K. 2019, ApJ, 883, 137

Rakshit, S., \& Woo, J.-H. 2018, ApJ, 865, 5

Rakshit, S., Woo, J.-H., Gallo, E., et al. 2019, ApJ, 886, 93

Rakshit, S., Stalin, C. S., \& Kotilainen, J. 2020, ApJS, 249, 17

Richards, J. L., Max-Moerbeck, W., Pavlidou, V., et al. 2011, ApJS, 194, 29

Rodríguez-Pascual, P. M., Alloin, D., Clavel, J., et al. 1997, ApJS, 110, 9

Schlafly, E. F., \& Finkbeiner, D. P. 2011, ApJ, 737, 103

Schmidt, G. D., Stockman, H. S., \& Smith, P. S. 1992, ApJ, 398, L57

Shakura, N. I., \& Sunyaev, R. A. 1973, A\&A, 500, 33

Shaw, M. S., Romani, R. W., Cotter, G., et al. 2012, ApJ, 748, 49

Shen, Y. 2013, Bull. Astron. Soc. India, 41, 61

Shen, Y., Horne, K., Grier, C. J., et al. 2016, ApJ, 818, 30

Sikora, M., Begelman, M. C., \& Rees, M. J. 1994, ApJ, 421, 153

Smith, P. S., Montiel, E., Rightley, S., et al. 2009, 2009 Fermi Symposium, eConf Proceedings C091122 [arXiv:0912.3621]

Tadhunter, C. N., Morganti, R., di Serego Alighieri, S., Fosbury, R. A. E., \& Danziger, I. J. 1993, MNRAS, 263, 999

Tavecchio, F., Maraschi, L., Ghisellini, G., et al. 2000, ApJ, 543, 535

Thompson, D. J., Djorgovski, S., \& de Carvalho, R. 1990, PASP, 102, 1235

Urry, C. M., \& Padovani, P. 1995, PASP, 107, 803

von Neumann, J. 1941, Ann. Math. Statist., 12, 367

Wandel, A., Peterson, B. M., \& Malkan, M. A. 1999, ApJ, 526, 579

Wang, J.-M., Qiu, J., Du, P., \& Ho, L. C. 2014, ApJ, 797, 65

Welsh, W. F. 1999, PASP, 111, 1347

White, R. J., \& Peterson, B. M. 1994, PASP, 106, 879

Whiting, M. T., Webster, R. L., \& Francis, P. J. 2001, MNRAS, 323, 718

Woo, J.-H., Yoon, Y., Park, S., Park, D., \& Kim, S. C. 2015, ApJ, 801, 38

Wood, M., Caputo, R., Charles, E., et al. 2017, PoS, ICRC2017, 824

Xie, G. Z., Liu, H. T., Cha, G. W., et al. 2005, AJ, 130, 2506

Zajaček, M., Czerny, B., Martinez-Aldama, M. L., et al. 2020, ApJ, 896, 146

Zhang, Z.-X., Du, P., Smith, P. S., et al. 2019, ApJ, 876, 49

Zu, Y., Kochanek, C. S., \& Peterson, B. M. 2011, ApJ, 735, 80

Zu, Y., Kochanek, C. S., Kozłowski, S., \& Udalski, A. 2013, ApJ, 765, 106 
Appendix A: Time series analysis of simulated light curves
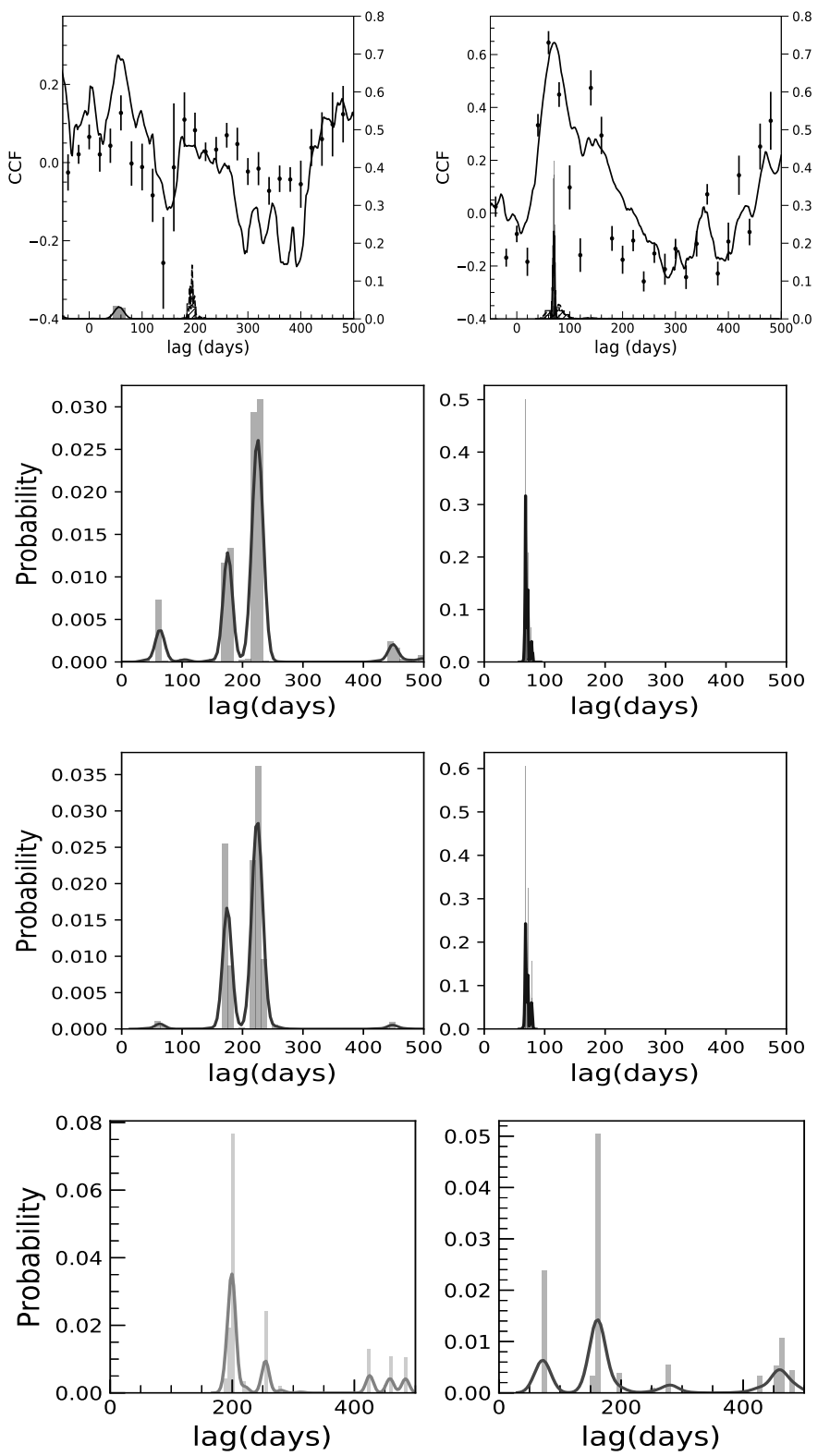

Fig. A.1. From top to bottom: lag probability distributions obtained from the CCF (ICCF and DCF), von Neumann, Bartels, and JAVELIN methods for the mock continuum vs. mock line light curve with a delay of 200 days (left) and 70 days (right). The lag probability distribution (histogram), along with the smoothed kernel density distribution, is shown in each panel.
To study the impact of seasonal gaps on the lag measurement, the DRW model implemented in JAVELIN was used to construct a mock continuum light curve that has similar characteristics as the observed $f_{5100}$ light curve of PKS 1510 . Then two mock line light curves were constructed with a lag of 70 and 200 days. The light curves were down-sampled to have the same time sampling as the observed light curves, therefore mimicking the observed time axis. The time series analysis methods (ICCF, DCF, von Neumann, Bartels, and JAVELIN) were then used on the mock continuum and line light curves to recover the input time lag. The results are shown in Fig. A.1 and given in Table A.1.

Table A.1. Time delay analysis results on mock light curves.

\begin{tabular}{lcc}
\hline \hline Method & \multicolumn{2}{c}{ Lag } \\
\hline Input lag & 70 & $\begin{array}{c}200 \\
(\text { days }) \\
(\text { days })\end{array}$ \\
$(1)$ & $(2)$ & $(3)$ \\
\hline ICCF & $70.0_{-2.0}^{+1.9}$ & Lag not calculated as $r_{\max }<0.3$ \\
DCF & $79.7_{-20.2}^{+15.9}$ & Lag not calculated as $r_{\max }<0.3$ \\
von Neumann & $68.8_{-1.1}^{+3.6}$ & $217.5_{-46.4}^{+16.9}$ \\
Bartels & $69.3_{-1.7}^{+5.5}$ & $217.9_{-43.9}^{+11.9}$ \\
JAVELIN & $161.2_{-89.6}^{+1.6}$ & $200.4_{-5.5}^{+53.4}$ \\
\hline
\end{tabular}

Notes. Columns are as follows: (1) method used; (2) input lag 70 days; and (3) input lag 200 days between the mock continuum and mock line light curves. 